\title{
Overexpression of IncRNA AFAP1-AS1 as a diagnostic biomarker in non-small cell lung cancer
}

\author{
Sajjad Ghalib Ibrahim Alnajar ${ }^{1}$, Ali Rajabi ${ }^{1}$, Melika Maydanchi ${ }^{2}$, Samaneh Tayefeh Gholami ${ }^{1}$, Ali Saber ${ }^{2}$ and \\ Reza Safaralizadeh ${ }^{1 *}$ (1)
}

\begin{abstract}
Background: Long non-coding RNAs (IncRNAs) play important roles in lung tumorigenesis. Among different IncRNAs, overexpression of the IncRNA actin filament-associated protein 1-antisense RNA 1 (AFAP1-AS1) in lung tumors has been reported in different studies. In the current study, we aimed to investigate the potential value of IncRNA AFAP1-AS1 as a diagnostic biomarker in lung cancer. Ninety samples from patients with lung cancer were collected from Noor-E-Nejat hospital, Tabriz, Iran. The expression of AFAP1-AS1 was assessed using quantitative reverse transcriptase-PCR (qRT-PCR), followed by the ROC curve analysis to investigate the biomarker potency of AFAP1-AS1.
\end{abstract}

Results: Our results revealed an upregulation of AFAP1-AS1 in tumor samples as compared to the adjacent nontumor tissues. We found a significant positive association between AFAP1-AS1 expression and tumor size, as well as tumor stage.

Conclusions: Our results showed overexpression of AFAP1-AS1 and its capacity as a diagnostic biomarker in lung cancer.

Keywords: LncRNAs, AFAP1-AS1, Diagnostic biomarker, Lung cancer, NSCLC

\section{Background}

Cancer is one of the most important health burdens and the second cause of death worldwide [1]. Over 1.8 million new cases and more than 600,000 deaths are estimated by the end of 2020 worldwide. Lung cancer is the second most common malignancy after breast cancer in women and prostate cancer [2,3]. It has been shown that tobacco smoking is one of the main risk factor of lung cancer susceptibility [4]. However, considering the heterogeneous nature of lung cancer, recent studies demonstrated that lung cancer incident is increasing in East Asia [5]. Lung cancer in never smokers may be associated with genetic and epigenetic profiles and with environmental

\footnotetext{
*Correspondence: Safaralizadeh@tabrizu.ac.ir

${ }^{1}$ Department of Animal Biology, Faculty of Natural Sciences, University of Tabriz, Tabriz, Iran

Full list of author information is available at the end of the article
}

factors including pollution, second-hand smoking [6]. Lung cancer is classified into two main histological subtypes, i.e., small cell lung cancer (SCLC) and non-small cell lung cancer (NSCLC); the latter with $80-85 \%$ of all cases is the most prevalent subtype [3, 7]. Also, NSCLC is further subdivided into lung squamous cell carcinoma (LUSC) and lung adenocarcinoma (LUAD), in which the first subtype is more common histology in never smokers and the latter one is more associated with smokers [8]. As a genetic susceptibility factor in lung cancer, non-coding regions of genome play a vital role in initiation and progression [9]. Long non-coding RNAs (lncRNAs) are non-coding endogenous RNAs with longer than 200 nucleotide length [10]. LncRNAs take part in a wide range of cellular processes, and their aberrant expression plays crucial roles in human malignancies [11, 12]. Several lncRNA aberrations are associated with cancer 
progression, invasion and metastasis [13]. For instance, the potential of lncRNAs MALAT1 (in prostate cancer), $M V I H$ (in hepatocellular carcinoma) and FENDRR (in gastric cancer) as diagnostic and diagnostic markers has been shown previously [14-16]. Lnc-RNAs exert their functions by affecting expression and post-translational modification of crucial proteins in normal cell development processes [17]. Align with these studies, the role of AFAP1-AS1 as a diagnostic biomarker in different cancer types has been shown. Thus, further investigations on AFAP1-AS1 expression can help to verify its potency as a diagnostic biomarker in cancer. Several studies have shown the role of IncRNAs in NSCLC progression and metastasis. Thus, lncRNAs are also of great importance to be used as diagnostic markers in different lung cancer subtypes $[18,19]$. For example, overexpression of lncRNA IGFBP4-1 was positively correlated with TNM staging and lung cancer susceptibility [20]. Furthermore, upregulation of other lncRNAs such as MIR4435-2HG causing transactivation of $\beta$-catenin, MALAT1 via inducing STAT3 activation, $D A N C R$ through sequestering miR-216a, UCA1 by targeting ERBB4 and lncRNA H19 by affecting epithelial to mesenchymal transition (EMT) process has been shown as key players of tumor progression and drug resistance in NSCLC [21-25]. LncRNA AFAP1-AS1 with 6,810 bp length is mapped to 4p16.1 and was first discovered in 2013 in Barrett's esophagus and esophageal adenocarcinoma [26]. It is transcribed in an antisense fashion and has overlap with AFAP1 gene [27]. Upregulation of AFAP1-AS1 is involved in lung tumorigenesis and metastasis in vitro [28-30]. AFAP1$A S 1$ has a critical function in cancer development, and it has the potential to be used as a diagnostic biomarker and therapeutic target [31]. Overexpression of AFAP1$A S 1$ is associated with poor clinical outcome in esophageal adenocarcinoma, pancreatic carcinoma, breast and lung cancer [32]. In addition, AFAP1-AS1 overexpression is associated with poor prognosis in NSCLC patients [11, 33].

The objective of the current study was to determine AFAP1-AS1 expression levels in NSCLC tumors compared to non-tumor tissues. We also aimed to study the association between AFAP1-AS1 expression and clinicopathological characteristics including smoking habits, gender, age, disease stage, tumor size and differentiation. Furthermore, AFAP1-AS1 value as a feasible and informative diagnostic biomarker was investigated.

\section{Methods}

\section{Sample collection}

Ninety NSCLC tumor and adjacent non-tumor tissues were obtained from Noor-E-Nejat hospital, Tabriz, Iran. Written informed consent was obtained from all participants. The study was approved by the Medical Ethic Committee of Tabriz University. This study was conducted according the Declaration of Helsinki and was in concordance with Good Clinical Practice guidelines. The histopathologic characteristics of the samples were evaluated and characterized by an experienced pathologist. The inclusion criteria for the patients in the current study were set to having NSCLC in patients admitted to the Noor-E-Nejat hospital, Tabriz, Iran. The exclusion criteria were absence of familial history of any cancer and alcohol consumption. Based on these criteria, totally, 90 patients were selected.

\section{RNA isolation, CDNA synthesis and qRT-PCR}

Total RNA was isolated using TRIZOL RNA extraction kit (Invitrogen, Massachusetts, USA) based on the manufacturer's protocol. DNaseI (GeneAll, Seoul, Korea) was applied for the elimination of DNA contamination. NanoDrop (Thermo Fisher scientific Nanodrop 2000, CA, USA) and $2 \%(\mathrm{v} / \mathrm{w})$ agarose gel electrophoresis were used to assess quantity and quality of RNA samples, respectively.

cDNA Synthesis was carried out using Takara kit (TaKaRa, Kusatsu, Japan) according to the manufacturer's instruction. Approximately, $100 \mathrm{ng}$ of cDNA was used for AFAP1-AS1 amplification by LightCycler ${ }^{\circledR} 96$ Real-Time PCR (Roche Molecular Systems, Inc., Pleasanton, CA, USA) using SYBR Green Master Mix (2x) (Amplicon, Odense, Denmark)., following primers were obtained from previously published study [39] for amplification: forward 5'-AGCCTGTTGAATCAGCCAACT$3^{\prime}$ and reverse 5'-GGTTCATACCAGCCCTGTCC- ${ }^{\prime}$. To normalize the expression of target gene, $\beta$-actin was amplified as housekeeping gene using following primers: forward $5^{\prime}$-AGAGCTACGAGCTGCCTGAC-3' and reverse $5^{\prime}$-AGCACTGTCTTGGCGTACAG- $3^{\prime}$. The cycle threshold $(\mathrm{Ct})$ was measured and difference between expression of AFAP1-AS1 and $\beta$-actin was defined as $\triangle \mathrm{Ct}$. To determine difference between the expression of AFAP1-AS1 and $\beta$-actin, $2^{-\Delta \mathrm{Ct}}$ value was calculated for each sample in tumor and corresponding non-tumor samples. All assays were performed in triplicate.

\section{Receiver operating characteristic (ROC)}

The ROC curve analysis was done to assess sensitivity and specificity of AFAP1-AS1 as a diagnostic biomarker in NSCLC.

\section{Statistical analysis}

Mann-Whitney test was used to compare differences in the expression of AFAP1-AS1 between tumor and nontumor samples. Association between AFAP1-AS1 expression and clinicopathological parameters was assessed 
using student's $t$ test and one-way ANOVA. The $t$ test was applied when the data were normally distributed, and Mann-Whitney test was performed otherwise. Statistical analysis was done using SPSS version 24 and GraphPad Prism 8. P values less than 0.05 were considered as significant.

\section{Results}

\section{Patients}

A total number of ninety NSCLC patients were included in the study. The majority of patients $(57 \%)$ were nonsmoker and $43 \%$ were smoker. Sixty-two percent (50/90) were below 55 years and 38\% (40/90) were older than 55 years. The main proportion of patients had their right side of their lung involved (64\%) and the remaining had their left side involved. Thirty-five (72\%) out of ninety patients were men and 25 (28/90) were women. Regarding tumor size, $72 \%$ (65/90) of patients had a tumor larger than $5 \mathrm{~cm}$ and the remaining had a tumor less than $5 \mathrm{~cm}$. Forty-two percent (47/90) of patients had poor and 38\% (43/90) had moderate to highly differentiated tumor. The half of patients had stage I/II and the other half had stage III/IV NSCLC.

\section{AFAP1-AS1 expression levels}

The expression of AFAP1-AS1 was significantly higher ( $p$ value $<0.0001$ ) in tumor samples as compared to the corresponding non-tumor tissues (Fig. 1). We observed a significant positive association between AFAP1-AS1 expression and tumor size $(\mathrm{p}=0.015)$. In addition, AFAP1-AS1 mean expression was significantly higher in stage III/IV group as compared to stage I/II group $(\mathrm{p}=0.019)$ (Table 1). However, we did not find any other significant association between AFAP1-AS1 expression levels and other clinicopathological features including

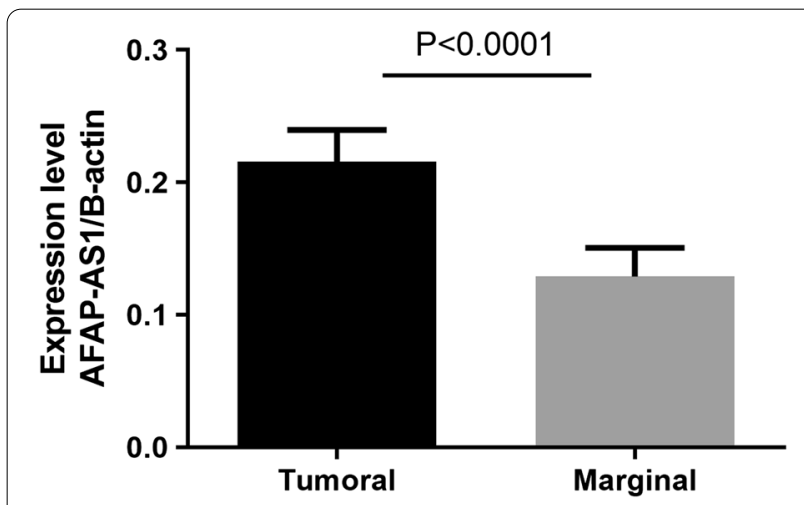

Fig. 1 The expression level of the Inc-AFAP1-AS1 between lung tumor and adjacent non-tumor tissues
Table 1 Association between IncRNA AFAP1-AS1 expression and clinicopathological characteristics in NSCLC patients

\begin{tabular}{|c|c|c|c|}
\hline Clinical parameter & No. of cases (\%) & $\begin{array}{l}\text { Mean } \\
\text { expression } \\
\left(2^{-\Delta \Delta C t}\right)\end{array}$ & $P$-value \\
\hline Smoking & & & 0.427 \\
\hline Yes & $39(43)$ & 14.12 & \\
\hline No & $51(57)$ & 13.35 & \\
\hline Age (Year) & & & 0.643 \\
\hline$\leq 55$ & $50(62)$ & 12.16 & \\
\hline$>55$ & $40(38)$ & 15.59 & \\
\hline Side of involvement & & & 0.993 \\
\hline Right & $58(64)$ & 13.28 & \\
\hline Left & $32(36)$ & 14.41 & \\
\hline Gender & & & 0.797 \\
\hline Male & $65(72)$ & 14.62 & \\
\hline Female & $25(28)$ & 11.26 & \\
\hline Tumor size (cm) & & & 0.015 \\
\hline$\geq 5$ & $64(72)$ & 15.43 & \\
\hline$<5$ & $26(28)$ & 9.40 & \\
\hline Lymph metastasis & & & 0.686 \\
\hline No & $42(47)$ & 13.57 & \\
\hline Yes & $48(53)$ & 13.78 & \\
\hline Differentiation & & & 0.728 \\
\hline Poor & $42(47)$ & 12.29 & \\
\hline Moderate/high & $48(53)$ & 14.90 & \\
\hline \multicolumn{4}{|l|}{ Stage } \\
\hline$|/| \mid$ & $45(50)$ & 9.98 & 0.019 \\
\hline III/IV & $45(50)$ & 17.39 & \\
\hline
\end{tabular}

The bold-face values are corresponding to the $P$ values $<0.05$, therefore statistically significant

smoking status, age, side of involvement, gender, lymph node metastasis and tumor differentiation.

\section{AFAP1-AS1 expression as diagnostic biomarker}

The ROC curve was plotted to assess the sensitivity and specificity of AFAP1-AS1 as a diagnostic biomarker (Fig. 2). The area under curve (AUC) was 0.6779 (CI 95\%, 0.5294 to 0.8263 ). Sensitivity and specificity of the biomarker were determined as $58 \%$ and $73 \%$, respectively, with the cutoff value of 9.909 .

\section{Discussion}

In the current study, we investigated the expression of lncRNA AFAP1-AS1 in lung tumors. We found a significant overexpression in lung tumors as compared to the matched non-tumor tissues. In addition, our analysis revealed a significant association between upregulated AFAP1-AS1 levels and tumor size, as well as the stage of NSCLC. 


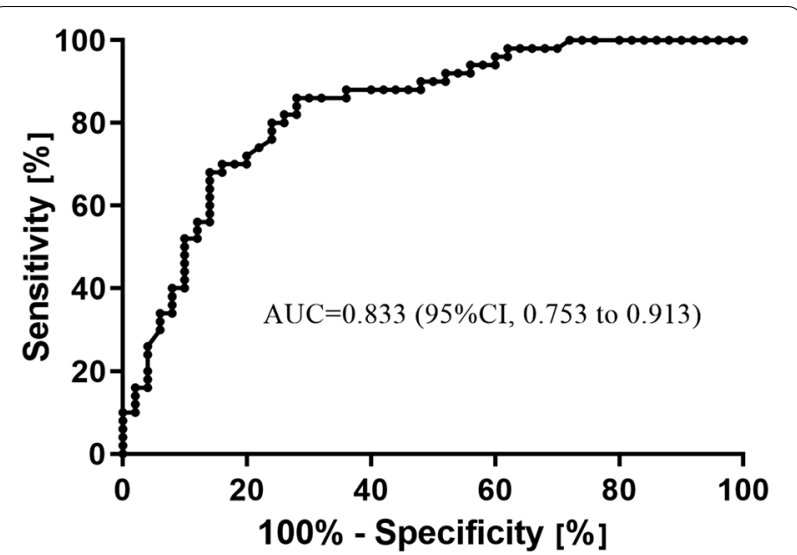

Fig. 2 The receiver operating characteristic $(\mathrm{ROC})$ curve analysis of AFAP1-AS1. The area under curve (AUC) is 0.6779 with $95 \%$ $\mathrm{Cl}=0.5294-0.8263$

Several gene mutations, copy number variations and epigenetic alterations are involved in cancer progression and distant metastasis. Due to the need for identification of novel therapeutic and diagnostic molecules, the role of lncRNAs in tumorigenesis has been highlighted in recent years [34]. For instance, upregulation of lncRNA H19 in lung cancer cells (A549) contributes to cell migration, invasion and EMT through regulating miR-484 affecting downstream pathways JNK and ROCK2 [25]. LncRNA $U C A 1$ exerts its oncogenic function via regulating miR193a-3p by a competitively 'sponging' mechanism which targets ERBB4 in lung cancer cells [24]. Furthermore, overexpression of other lncRNAs, such as IGFBP4 and $D A N C R$, in lung cancer provides supporting evidence for their oncogenic role [20,23].

AFAP1-AS1 is involved in cancer progression via affecting EMT process [35], modulating expression of several small GTPase members and aberrations in actin cytokeratin signaling pathway [36], regulating Rho/Rac pathway, downregulating C-myc and cycling D1 [26, 37], RhoC, p38MAPK, ROCK1 and Twist1 [29, 38]. Zhuang et al. (2017) showed AFAP1-AS1 upregulation in lung adenocarcinoma as compared to non-tumor tissues. In addition, AFAP1-AS1 downregulation was significantly associated with higher survival rate; thus, this lncRNA may serve as an effective diagnostic biomarker [28].

We found a positive correlation between AFAP1-AS1 expression and tumor size, as well as the stage of disease. Furthermore, the ROC curve analysis, by plotting the expression level of AFAP1-AS1 in stage I/II compared to stage III/IV, revealed AFAP1-AS1 potential as an acceptable diagnostic biomarker. Our results were concordant with previous studies where AFAP1-AS1 expression appeared to be a valuable diagnostic marker in patients with NSCLC [11, 30, 33]. Moreover, Li et al. [33] showed the biomarker potency of circulating AFAP1-AS1 in discriminating between NSCLC patients from healthy people.

\section{Conclusion}

In conclusion, IncRNA AFAP1-AS1 was upregulated in NSCLC tumors as compared to non-tumor samples. Our analysis revealed that AFAP1-AS1 might serve as a diagnostic biomarker in NSCLC. As we did not investigate underlying mechanisms by which AFAP1-AS1 exerts its biological function, more profound studies are warranted.

\section{Abbreviations}

NSCLC: Non-small cell lung cancer; SCLC: Small cell lung cancer; EMT: Epithelial to mesenchymal transition; ROC: Receiver operating characteristic; AUC: Area under curve; Ct: Cycle threshold.

\section{Acknowledgements}

Not applicable.

\section{Authors' contributions}

AS and RS designed the study. AR and SA performed experimental work. AR, $S A, M M, S T G, A S$ and RS analyzed the data. AR and SA wrote the manuscript with significant input from AS, MM and RS. AS edited the final draft and provided technical advice. RS supervised the project. All authors have read and approved the manuscript.

\section{Funding}

Not applicable.

\section{Availability of data and material}

The datasets used and/or analyzed during the current study are available from the corresponding author on reasonable request.

\section{Declarations}

\section{Ethics approval and consent to participate}

The study was approved by the Medical Ethic Committee of Tabriz University (with the approval number of IR.TABRIZU.REC.1398.015). Written consent was obtained from all of the participants. This study was conducted according the Declaration of Helsinki and was in concordance with Good Clinical Practice guidelines.

\section{Consent for publication}

Not applicable.

\section{Competing interests}

The authors declare that they have no competing interests.

\section{Author details}

${ }^{1}$ Department of Animal Biology, Faculty of Natural Sciences, University of Tabriz, Tabriz, Iran. ${ }^{2}$ Zimagene Medical Genetics Laboratory, Avicenna St., Hamedan, Iran.

Received: 20 April 2021 Accepted: 13 August 2021

Published online: 05 October 2021 


\section{References}

1. Henley SJ, Ward EM, Scott S, Ma J, Anderson RN, Firth AU et al (2020) Annual report to the nation on the status of cancer, part i: national cancer statistics. Cancer 126(10):2225-2249

2. Siegel RL, Miller KD, Jemal A (2020) Cancer statistics, 2020. CA Cancer J Clin 70(1):7-30

3. Ferronika P, van den Bos H, Taudt A, Spierings DCJ, Saber A, Hiltermann TJN et al (2017) Copy number alterations assessed at the single-cell level revealed mono- and polyclonal seeding patterns of distant metastasis in a small-cell lung cancer patient. Ann Oncol 28(7):1668-1670

4. Khuder SA (2001) Effect of cigarette smoking on major histological types of lung cancer: a meta-analysis. Lung Cancer 31(2-3):139-148

5. Sun S, Schiller JH, Gazdar AF (2007) Lung cancer in never smokers-a different disease. Nat Rev Cancer 7(10):778-790

6. Vineis P, Airoldi L, Veglia F, Olgiati L, Pastorelli R, Autrup H et al (2005) Environmental tobacco smoke and risk of respiratory cancer and chronic obstructive pulmonary disease in former smokers and never smokers in the EPIC prospective study. BMJ 330(7486):277

7. Saber A, Hiltermann TJN, Kok K, Terpstra MM, de Lange K, Timens W et al (2017) Mutation patterns in small cell and non-small cell lung cancer patients suggest a different level of heterogeneity between primary and metastatic tumors. Carcinogenesis 38(2):144-151

8. Molina JR, Yang P, Cassivi SD, Schild SE, Adjei AA (2008) Non-small cell lung cancer: epidemiology, risk factors, treatment, and survivorship. Mayo Clin Proc 83(5):584-594

9. Yang Y-R, Zang S-Z, Zhong C-L, Li Y-X, Zhao S-S, Feng X-J (2014) Increased expression of the IncRNA PVT1 promotes tumorigenesis in non-small cell lung cancer. Int J Clin Exp Pathol 7(10):6929

10. Ji D, Zhong $X$, Jiang $X$, Leng $K, X u Y$, Li Z et al (2018) The role of long noncoding RNA AFAP1-AS1 in human malignant tumors. Pathol Res Pract 214(10):1524-1531

11. Deng J, Liang Y, Liu C, He S, Wang S (2015) The up-regulation of long noncoding RNA AFAP1-AS1 is associated with the poor prognosis of NSCLC patients. Biomed Pharmacother 75:8-11

12. Rajabi A, Riahi A, Shirabadi-Arani H, Moaddab Y, Haghi M, Safaralizadeh R (2020) Overexpression of HOXA-AS2 LnCRNA in patients with gastric cancer and its association with helicobacter pylori infection. J Gastrointest Cancer

13. Liu Q, Huang J, Zhou N, Zhang Z, Zhang A, Lu Z et al (2013) LncRNA loc285194 is a p53-regulated tumor suppressor. Nucleic Acids Res 41(9):4976-4987

14. Ren S, Wang F, Shen J, Sun Y, Xu W, Lu J et al (2013) Long non-coding RNA metastasis associated in lung adenocarcinoma transcript 1 derived miniRNA as a novel plasma-based biomarker for diagnosing prostate cancer. Eur J Cancer 49(13):2949-2959

15. Yuan SX, Yang F, Yang Y, Tao QF, Zhang J, Huang G et al (2012) Long noncoding RNA associated with microvascular invasion in hepatocellular carcinoma promotes angiogenesis and serves as a predictor for hepatocellular carcinoma patients' poor recurrence-free survival after hepatectomy. Hepatology 56(6):2231-2241

16. Xu T-P, Xia R, Liu X-X, Sun M, Yin L, Chen W-M et al (2014) Decreased expression of the long non-coding RNA FENDRR is associated with poor prognosis in gastric cancer and FENDRR regulates gastric cancer cell metastasis by affecting fibronectin1 expression. J Hematol Oncol. 7(1):63

17. Qi P, Du X (2013) The long non-coding RNAs, a new cancer diagnostic and therapeutic gold mine. Mod Pathol 26(2):155-165

18. Lu T, Wang Y, Chen D, Liu J, Jiao W (2018) Potential clinical application of IncRNAs in non-small cell lung cancer. Onco Targets Ther 11:8045-8052

19. Wei J, Rybczynska AA, Meng P, Terpstra M, Saber A, Sietzema J et al (2020) An all-in-one transcriptome-based assay to identify therapy-guiding genomic aberrations in nonsmall cell lung cancer patients. Cancers (Basel) 12(10):2843

20. Yang B, Zhang L, Cao Y, Chen S, Cao J, Wu D et al (2017) Overexpression of IncRNA IGFBP4-1 reprograms energy metabolism to promote lung cancer progression. Mol Cancer 16(1):154

21. Qian H, Chen L, Huang J, Wang X, Ma S, Cui F et al (2018) The IncRNA MIR4435-2HG promotes lung cancer progression by activating $\beta$-catenin signalling. J Mol Med 96(8):753-764
22. Fang Z, Chen W, Yuan Z, Liu X, Jiang H (2018) LncRNA-MALAT1 contributes to the cisplatin-resistance of lung cancer by upregulating MRP1 and MDR1 via STAT3 activation. Biomed Pharmacother 101:536-542

23. Zhen Q, Gao L-N, Wang R-F, Chu W-W, Zhang Y-X, Zhao X-J et al (2018) LncRNA DANCR Promotes Lung Cancer by Sequestering miR-216a. Cancer Control 25(1):1073274818769849

24. Nie W, Ge H-J, Yang X-Q, Sun X, Huang H, Tao X et al (2016) LnCRNA-UCA1 exerts oncogenic functions in non-small cell lung cancer by targeting miR-193a-3p. Cancer Lett 371(1):99-106

25. Zhang Q, Li X, Li X, Li X, Chen Z (2018) LnCRNA H19 promotes epithelialmesenchymal transition (EMT) by targeting miR-484 in human lung cancer cells. J Cell Biochem 119(6):4447-4457

26. Wu W, Bhagat TD, Yang X, Song JH, Cheng Y, Agarwal R et al (2013) Hypomethylation of noncoding DNA regions and overexpression of the long noncoding RNA, AFAP1-AS1 Barrett's esophagus and esophageal adenocarcinoma. Gastroenterology 144(5):956-966

27. Zhang F, Li J, Xiao H, Zou Y, Liu Y, Huang W (2018) AFAP1-AS1: A novel oncogenic long non-coding RNA in human cancers. Cell Prolif. 51(1):e12397

28. Zhuang Y, Jiang H, Li H, Dai J, Liu Y, LiY et al (2017) Down-regulation of long non-coding RNA AFAP1-AS1 inhibits tumor cell growth and invasion in lung adenocarcinoma. Am J Transl Res 9(6):2997

29. Lu X, Zhou C, Li R, Liang Z, Zhai W, Zhao L et al (2016) Critical role for the long non-coding RNA AFAP1-AS1 in the proliferation and metastasis of hepatocellular carcinoma. Tumor Biol 37(7):9699-9707

30. Zeng Z, Bo H, Gong Z, Lian Y, Li X, Li X et al (2016) AFAP1-AS1, a long noncoding RNA upregulated in lung cancer and promotes invasion and metastasis. Tumor Biol 37(1):729-737

31. Ji D, Zhong $X$, Jiang $X$, Leng $K, X u Y$, Li Z et al (2018) The role of long noncoding RNA AFAP1-AS1 in human malignant tumors. Pathol Res Pract 214(10):1524-1531

32. Liu F-T, Xue Q-Z, Zhu P-Q, Luo H-L, Zhang Y, Hao T (2016) Long noncoding RNA AFAP1-AS1, a potential novel biomarker to predict the clinical outcome of cancer patients: a meta-analysis. Onco Targets Ther 9:4247-4254

33. Li W, Li N, Kang X, Shi K (2017) Circulating long non-coding RNA AFAP1AS1 is a potential diagnostic biomarker for non-small cell lung cancer. Clin Chim Acta 475:152-156

34. Castillo J, Stueve TR, Marconett CN (2017) Intersecting transcriptomic profiling technologies and long non-coding RNA function in lung adenocarcinoma: discovery, mechanisms, and therapeutic applications. Oncotarget 8(46):81538

35. Han X, Wang L, Ning Y, Li S, Wang Z (2016) Long non-coding RNA AFAP1AS1 facilitates tumor growth and promotes metastasis in colorectal cancer. Biol Res 49(1):36

36. Bo H, Gong Z, Zhang W, Li X, Zeng Y, Liao Q et al (2015) Upregulated long non-coding RNA AFAP1-AS1 expression is associated with progression and poor prognosis of nasopharyngeal carcinoma. Oncotarget 6(24):20404-20418

37. Lu X, Zhou C, Li R, Deng Y, Zhao L, Zhai W (2017) Long noncoding RNA AFAP1-AS1 promoted tumor growth and invasion in cholangiocarcinoma. Cell Physiol Biochem 42(1):222-230

38. Shi D, Wu F, Mu S, Hu B, Zhong B, Gao F et al (2019) LncRNA AFAP1-AS1 promotes tumorigenesis and epithelial-mesenchymal transition of osteosarcoma through RhoC/ROCK1/p38MAPK/Twist1 signaling pathway. J Exp Clin Cancer Res 38(1):375

39. Yang ZT, An F, Hu JD, Zhao WH (2019) Long noncoding RNA AFAP1-AS1 accelerates the proliferation and metastasis of prostate cancer via inhibiting RBM5 expression. Eur Rev Med Pharmacol Sci 23(8):3284-3290

\section{Publisher's Note}

Springer Nature remains neutral with regard to jurisdictional claims in published maps and institutional affiliations. 